\title{
Structural Health Monitoring
}

\section{Broadband nonlinear elastic wave modulation spectroscopy for damage detection in composites}

\begin{tabular}{|c|c|}
\hline Journal: & Structural Health Monitoring \\
\hline Manuscript ID & SHM-20-0481.R1 \\
\hline Manuscript Type: & Original Manuscript \\
\hline $\begin{array}{r}\text { Date Submitted by the } \\
\text { Author: }\end{array}$ & $n / a$ \\
\hline Complete List of Authors: & $\begin{array}{l}\text { Segers, Joost; Ghent University Faculty of Engineering and Architecture, } \\
\text { Metareials, Textiles and Chemical Engineering } \\
\text { Hedayatrasa, Saeid; Ghent University Faculty of Engineering and } \\
\text { Architecture, Metareials, Textiles and Chemical Engineering } \\
\text { Poelman, Gaétan; Ghent University Faculty of Engineering and } \\
\text { Architecture, Metareials, Textiles and Chemical Engineering } \\
\text { Van Paepegem, Wim; Ghent University Faculty of Engineering and } \\
\text { Architecture, Materials, Textiles and Chemical Engineering } \\
\text { Kersemans, Mathias; Ghent University Faculty of Engineering and } \\
\text { Architecture, Materials, Textiles and Chemical Engineering }\end{array}$ \\
\hline Keywords: & $\begin{array}{l}\text { Composites, Nonlinearity, Elastic wave modulation, Bandpower, } \\
\text { Scanning laser Doppler vibrometry, Non-destructive testing }\end{array}$ \\
\hline Abstract: & $\begin{array}{l}\text { A NDT procedure is proposed for damage detection in composites using } \\
\text { full wavefield measurement obtained using a laser Doppler vibrometer. } \\
\text { Vibrations are excited using two low-power piezoelectric actuators } \\
\text { leading to nonlinear elastic wave modulation at the defect. One actuator } \\
\text { is supplied with a broadband chirp signal and the other actuator is } \\
\text { supplied with a single-frequency sine signal. First, a time-frequency } \\
\text { filtering method is proposed to extract specific nonlinear components of } \\
\text { interest (e.g. second higher harmonic and first modulation sideband) } \\
\text { without the need for multiple excitation sequences. Next, damage maps } \\
\text { are constructed using broadband bandpower calculation of the filtered } \\
\text { nonlinear components. It is demonstrated that the modulation sidebands } \\
\text { provide an exclusive imaging of defect nonlinearity, and are not affected } \\
\text { by potential source nonlinearity. } \\
\text { The proposed damage map construction procedure is applied for various } \\
\text { carbon fiber reinforced polymer test specimens with different damage } \\
\text { features: (i) coupon with quasi static indentation damage, (ii) coupon } \\
\text { with artificial delaminations, (iii) bicycle frame with impact damage and } \\
\text { (iv) stiffened aircraft panel with partially debonded stiffener. } \\
\text { The obtained results indicate the high performance of the developed } \\
\text { procedure for detection of various defect types in curved and/or stiffened } \\
\text { CFRP components. }\end{array}$ \\
\hline
\end{tabular}


2

3

4

5

6

7

8

9

10

11

12

13

14

15

16

17

18

19

20

21

22

23

24

25

26

27

28

29

30

31

32

33

34

35

36

37

38

39

40

41

42

43

44

45

46

47

48

49

50

51

52

53

54

55

56

57

58

59

60

\section{SCHOLARONE ${ }^{\text {" }}$ \\ Manuscripts}

http://mc.manuscriptcentral.com/shmij 


\title{
Broadband nonlinear elastic wave modulation spectroscopy for damage detection in composites
}

\author{
Joost Segers ${ }^{1 \#}$, Saeid Hedayatrasa ${ }^{1,2}$, Gaétan Poelman¹, Wim Van Paepegem ${ }^{1}$ \\ and Mathias Kersemans ${ }^{1 \#}$ \\ ${ }^{1}$ Mechanics of Materials and Structures (UGent-MMS), Department of Materials, Textiles and \\ Chemical Engineering (MaTCh), Ghent University, Technologiepark-Zwijnaarde 46, 9052 Zwijnaarde, \\ Belgium \\ \#Corresponding authors: Joost.Segers@ugent.be, Mathias.Kersemans@ugent.be \\ ${ }^{2}$ SIM Program M3 DETECT-IV, Technologiepark-Zwijnaarde 48, B-9052 Zwijnaarde, Belgium
}

\begin{abstract}
A NDT procedure is proposed for damage detection in composites using full wavefield measurement obtained using a laser Doppler vibrometer. Vibrations are excited using two low-power piezoelectric actuators leading to nonlinear elastic wave modulation at the defect. One actuator is supplied with a broadband chirp signal and the other actuator is supplied with a single-frequency sine signal. First, a time-frequency filtering method is proposed to extract specific nonlinear components of interest (e.g. second higher harmonic and first modulation sideband) without the need for multiple excitation sequences. Next, damage maps are constructed using broadband bandpower calculation of the filtered nonlinear components. It is demonstrated that the modulation sidebands provide an exclusive imaging of defect nonlinearity, and are not affected by potential source nonlinearity.

The proposed damage map construction procedure is applied for various carbon fiber reinforced polymer test specimens with different damage features: (i) coupon with quasi static indentation damage, (ii) coupon with artificial delaminations, (iii) bicycle frame with impact damage and (iv) stiffened aircraft panel with partially debonded stiffener.

The obtained results indicate the high performance of the developed procedure for detection of various defect types in curved and/or stiffened CFRP components.
\end{abstract}

\section{Keywords}

Composites; Non-destructive testing (NDT); Nonlinearity; Elastic wave modulation; Bandpower; Scanning laser Doppler vibrometry

\section{Introduction}

Layered fiber reinforced polymer materials, or composites, are characterized by a high specific strength and stiffness. Because of this, composites are increasingly used in the automotive and aerospace industry. A drawback in the use of composite materials is related to the occurrence of internal damages or defects. In order to guarantee the safe operation of the composite parts, they have to be inspected for internal defects after production and at regular intervals during the operational lifetime.

Carbon fiber reinforced polymer (CFRP) components are often large and thin-walled which makes the use of vibrometric non-destructive testing (NDT) methods highly interesting. The frequency of vibration used for NDT based on guided waves is typically in the $\mathrm{kHz}$ range. The vibrations can be excited using surface-bonded piezoelectric transducers [1], air-coupled piezoelectric transducers [2] or using non-contact (scanning) pulsed lasers [3]. Sensing of the guided waves is possible using the same piezoelectric transducers $[1,4]$ or with a non-contact (scanning) laser Doppler vibrometer (S)LDV. The 
most accurate detection and localization of potential small damages in complex composite parts (in a baseline-free manner) is obtained for techniques which analyze the full wavefield measured using scanning laser excitation or SLDV sensing [5].

When an elastic wave interacts with a defect, the wavefield might get distorted depending on the characteristics of the elastic wave and the characteristics of the defect. In the last decades, multiple damage detection approaches were developed which focus on specific wave-defect interactions for the construction of a damage map [5].

First of all, the defect can change the local amplitude of vibration. Bending stiffness reducing defects, such as delaminations, result in an increased local vibrational amplitude. Further, if the wavelength of the wave matches the defect size, a further increase in vibrational amplitude can occur due to wave trapping. This phenomenon is referred to as energy (or mode) trapping [6, 7]. Energy-based damage map construction methods, such as weighted root mean square (WRMS) energy calculation [8-10], exploit this phenomenon and detect the defects as spots of increased vibrational intensity. At specific frequencies, the energy trapping is highly efficient leading to Local Defect Resonances (LDR) [11, 12] which can be detected in a broadband chirp response in an automated manner [13]. However, deep defects only result in a minor change of the local stiffness which makes their detection using these energy (or LDR) based approaches problematic [14].

Next, multiple methods were developed which exploit the local change in wavenumber at a defect [1519]. The local wavenumber estimation methods offer the additional advantage that the local wavenumber can be used for accurate estimation of the depth of the defect [19, 20]. However, also these methods suffer from limitations. The local wavenumber estimation requires measurement data on a grid of equidistance scan points, which is often challenging to achieve for complex or curved structural components. Also, in order to detect small defects, a very dense grid of scan points is required which increases the measurement time considerably. In addition, these wavenumber approaches lack the sensitivity to detect deep defects (i.e. defect's depth $>80 \%$ of sample's thickness) $[21,22]$.

Using specific filters in the wavenumber-frequency domain, it is possible to remove the vibrations that correspond to the Lamb modes travelling in damage-free material while retaining the vibrations attributed to defects. As an example, the incident wavefield can be removed using directional filtering, revealing the defect as the location of scattered waves [23-25]. Alternatively, mode or wavenumber filters can be used to remove vibrations related to specific modes or wavenumbers after which an improved damage map is obtained by WRMS energy calculation [26-28]. Current authors recently developed a broadband mode-removed WRMS approach, and demonstrated the accurate localization of small and deep production defects in a stiffened CFRP aircraft panel [29].

At last, the interaction of a defect with elastic waves can be nonlinear in nature. As such, defects can be detected by searching for nonlinear vibrational components in the measured wavefield. The nonlinear response of a defect is attributed to contact and friction at the defect's interfaces (i.e. contact acoustic nonlinearity) which generally requires a sufficiently high vibrational amplitude to be triggered [30]. As such, it is beneficial to match the excitation frequency with a frequency for which efficient energy trapping, or better LDR, occurs [11]. When a single source of vibrations is employed, the nonlinear elastic wave spectroscopy (NEWS) shows promising results in revealing the defect as a source of higher harmonic components [31-39]. Current authors showed that a backside delamination (i.e. defect's depth $>80 \%$ of the sample's thickness) acts as a secondary source of higher harmonics when the excitation frequency matches the defect's backside LDR frequency [40]. In order to increase the nonlinear response at the defect, a second excitation source can be added. The nonlinear response of the defect results in frequency mixing and the presence of modulation sidebands in the spectrogram. The study of this frequency modulation is referred to as nonlinear elastic wave modulation spectroscopy (NWMS) [41-47].

The NEWS and NWMS methods are promising for NDT as they show the best potential to detect small and deep defects but there are still some critical hurdles to be overcome: 
- The nonlinear response is only triggered in case of a sufficiently high vibrational amplitude at the defect. When low power actuators are used, this high vibrational amplitude is only reached for specific excitation frequencies (e.g. a LDR frequency), which requires a priori information on the defect properties [30].

- The nonlinear components of interest have to be extracted out of the measurement dataset. In case of a broadband excitation (e.g. a broadband chirp), the fast Fourier transform (FFT) cannot be used as the nonlinear components overlap with the linear response in the frequency domain. As a solution, often multiple excitation sequences are used and the corresponding measurements are combined with the aim to cancel out the linear response and retain only specific nonlinear components. As an example, the modulation sidebands can be extracted by combination of the results of three individual excitation sequences: (i) only chirp source activated, (ii) only sine source activated and (iii) both sources activated [48] or by combination of the results of two phase shifted excitations (i.e. phase symmetry analysis) [49]. However, the use of multiple excitation sequences increases the measurement time and complexity.

- The excitation sources can introduce nonlinear vibrational components on their own (i.e. source nonlinearity) which may dominate over the nonlinear components introduced by the defect.

In this study, we aim to overcome these remaining hurdles or limitation using well-chosen excitation signals and novel data processing techniques:

- Two excitation sources are used which are respectively excited with a broadband chirp signal and a single frequency sine signal. The broadband nature of the excitation makes sure that efficient energy trapping at the defect occurs, e.g. at the LDR frequency. This results in a strong vibrational activity of the defect, and as such triggers the defect's nonlinear behavior.

- Automated filtering in time-frequency domain is applied for the extraction of specific nonlinear components, such as higher harmonics and modulation sidebands, from the single broadband measurement.

- The origin of the extracted nonlinear components is investigated with the aim to exclude source nonlinearity, and to retain only defect nonlinearity.

- Broadband bandpower calculation is proposed for robust damage map construction.

The performance of the obtained damage maps is evaluated first for academic CFRP coupons with quasi-static indentation damage (QSID) and with artificial delaminations. Afterwards, the effectiveness of the proposed methods is verified using measurement results of complex industrial CRFP test specimens.

In section 2, the measurement procedure and the used test specimens are described. Section 3 outlines the developed NWMS methodology. The nonlinear component extraction and damage map construction is explained in detail using the measurement results of a CFRP coupon with QSID. In Section 4, the damage detection capability is investigated on three more CFRP test specimens: (i) a coupon with a shallow and a deep artificial delamination, (ii) a bicycle frame down tube with barely visible impact damage (BVID) and (iii) a stiffened aircraft panel with a partially debonded stiffener.

\section{Materials and Measurements}

Measurements are performed on four different CFRP test specimens (see Figure 1).

The first component (Figure 1 (a)) is a $150 \times 100 \times 5.5 \mathrm{~mm}^{3}$ coupon manufactured out of 24 layers of unidirectional carbon fiber according to a quasi-isotropic stacking sequence $[(45 / 0 /-45 / 90)]_{3 s}$. A quasistatic indentation is applied according to ASTM D6264 standard with $2.2 \mathrm{~mm}$ indentation displacement. The resulting quasi-static indentation damage (QSID) is highly similar to the damage which resulting from low velocity impact, i.e. BVID [50], as shown by the ultrasonic C-scan's time-offlight (TOF) image. The C-scan is performed in reflection mode using dynamic time gating. A focused 
transducer at $5 \mathrm{MHz}$ (H5M, General Electric) is employed. The backside of the specimen is inspected for damage (see Figure 1 (a)). Two identical piezoelectric actuators with diameter $12 \mathrm{~mm}$ and thickness $0.2 \mathrm{~mm}$ (Ekulit type EPZ-20MS64W) are bonded to the indented side using epoxy (Araldite rapid). One of these actuators is supplied with a linear chirp voltage signal from 1 to $200 \mathrm{kHz}$ at $65 \mathrm{~V}_{\mathrm{pp}}$ whereas the other actuator is supplied with a $100 \mathrm{~V}_{\mathrm{pp}}$ sine voltage signal. Three separate measurements are performed where the sine frequency is set at $50 \mathrm{kHz}, 63 \mathrm{kHz}$ and $70 \mathrm{kHz}$. This allows to investigate the effect of this sine frequency selection on the nonlinear response of the defect.

The second component (Figure 1 (b)) is a $290 \times 140 \times 2.5 \mathrm{~mm}^{3}$ CFRP coupon manufactured out of 8 layers of unidirectional carbon fiber according to a cross-ply stacking sequence $[(0 / 90)]_{2 s}$. Two artificial delaminations were introduced during the layup process using $20 \times 20 \mathrm{~mm}^{2}$ inserts made out of a double layer of $25 \mu \mathrm{m}$ thin brass foil. The inserts were placed between the $1^{\text {st }}$ and $2^{\text {nd }}$ layer, and between the $7^{\text {th }}$ and $8^{\text {th }}$ layer, resulting in a shallow (frontside) and a deep (backside) delamination, respectively. Again, two small piezoelectric actuators are bonded to the backside, this time using phenyl salicylate which has to advantage that the actuators can easily be removed afterwards by heating of the substance. One actuator is supplied with a linear chirp voltage from 10 to $125 \mathrm{kHz}$ at 65 $\mathrm{V}_{\mathrm{pp}}$ whereas the other actuator is supplied with a $30 \mathrm{kHz}$ sine voltage at $100 \mathrm{Vpp}$.

The third component is a bicycle frame (Figure 1 (c)). There is no information on the layup or thickness of the tubular CFRP sections. The down tube suffered an impact which introduced BVID. From a previous study, three small actuators were already bonded to the topside of the down tube using epoxy. The two actuators at the right are supplied with the chirp voltage signal (10 to 125 $\mathrm{kHz}, 65 \mathrm{~V}_{\mathrm{pp}}$ ) whereas the third actuator at the left is used for the sine excitation ( $30 \mathrm{kHz}$ and $50 \mathrm{kHz}$, $100 \mathrm{~V}_{\mathrm{pp}}$ ).

The fourth and last test specimen is an Airbus A320 vertical fin rib panel (Figure 1 (d)). The component's base plate measures $600 \times 200 \times 1.1 \mathrm{~mm}^{3}$ and contains three vertical stiffeners at the backside. The material layup is unknown. This component was scrapped by the manufacturer after damage was detected at the middle stiffener. The cause of the damage is unknown. The damage pattern is revealed using an ultrasonic $\mathrm{C}$-scan (reflection mode with dynamic time gating and $5 \mathrm{MHz}$ focused transducer) and consists out of a shallow delamination at the left side of the stiffener's fin and a larger debonding between the stiffener and base plate at the right side of the fin. The piezoelectric actuators are bonded to the backside using removable phenyl salicylate and are supplied with a chirp voltage signal (10 to $\left.125 \mathrm{kHz}, 65 \mathrm{~V}_{\mathrm{pp}}\right)$ and a sine voltage signal $\left(30 \mathrm{kHz}, 100 \mathrm{~V}_{\mathrm{pp}}\right)$, respectively.

In all cases, the chirp excitation signal is provided by the SLDV's built-in function generator which is then amplified through a Falco WMA-300 high voltage amplifier. The sine excitation signal is supplied by an external function generator (Tektronix AFG3021B) through an AR 150A100D power amplifier. The excitation characteristics are summarized in Table 1.

The $65 \mathrm{~V}_{\mathrm{pp}}$ and $100 \mathrm{~V}_{\mathrm{pp}}$ excitation voltage amplitudes for the chirp and sine actuators, respectively, result in a reliable and safe operation of the low power piezoelectric actuators. Using these voltages, the out-of-plane velocity amplitude at the defects goes up to $150 \mathrm{~mm} / \mathrm{s}$ at the LDR frequencies (i.e. strain amplitudes up to $10^{-7}$ ), which is sufficient to trigger contact acoustic nonlinearity. Higher voltage amplitudes result in higher vibrational amplitudes (at all frequencies) and can further enhance the nonlinear response of the defect. However, the employed small actuators may quickly degrade when an excessive voltage amplitude is applied.

The selected start $f_{\text {start }}$ and end $f_{\text {end }}$ frequencies of the chirp signal cover the broad frequency range where multiple LDR and energy trapping phenomena are expected $[6,7,14]$. The CFRP coupon with QSID is considerably thicker compared to the other three test specimens, resulting in the higher chirp end frequency. 


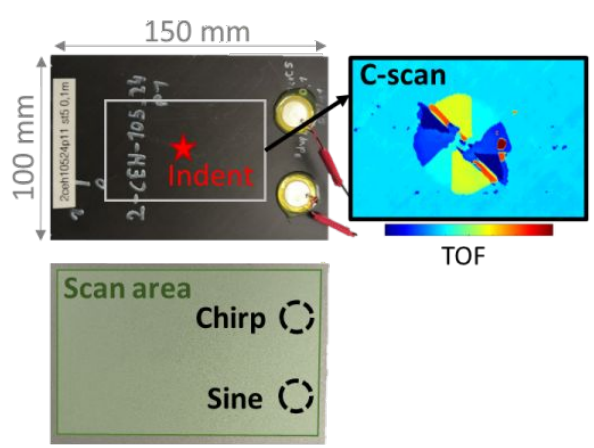

(a)

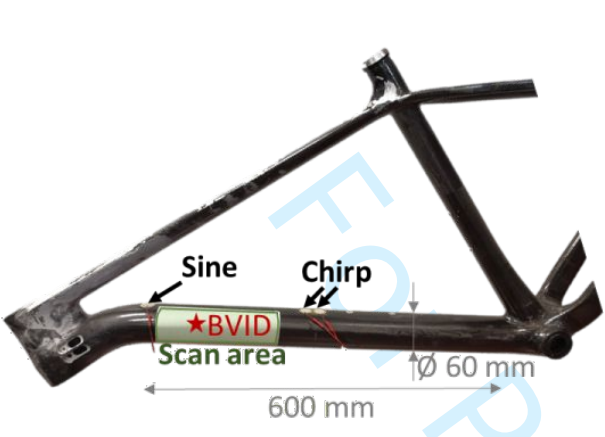

(c)

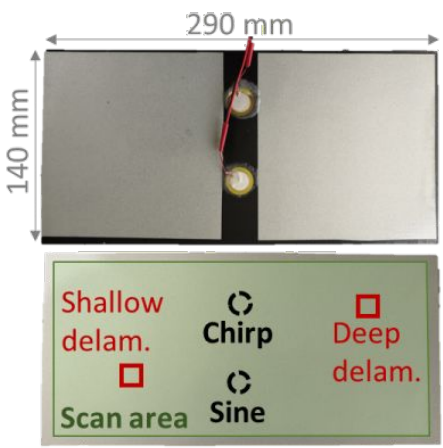

(b)

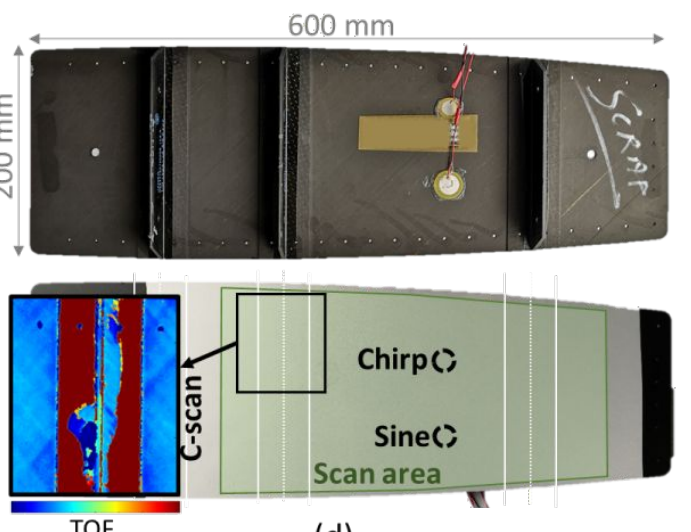

(d)

Figure 1: CFRP test specimens: (a) Quasi-isotropic coupon with QSID, (b) Cross-ply coupon with shallow and deep artificial delamination, (c) Bicycle frame with impacted down tube, (d) Stiffened aircraft panel with damage at the middle stiffener.

Table 1: Characteristics of excitation signals and SLDV data acquisition.

\begin{tabular}{|c|c|c|c|c|c|c|c|c|c|}
\hline \multirow[b]{2}{*}{ Component } & \multicolumn{3}{|c|}{ Chirp PZT } & \multicolumn{2}{|c|}{ Sine PZT } & \multicolumn{4}{|c|}{ SLDV } \\
\hline & $\begin{array}{c}\mathrm{f}_{\text {start }} \\
(\mathrm{kHz})\end{array}$ & $\begin{array}{c}\mathrm{f}_{\text {end }} \\
(\mathrm{kHz})\end{array}$ & Vpp & $\begin{array}{l}\mathrm{f}_{\text {sine }} \\
(\mathrm{kHz})\end{array}$ & Vpp & $\begin{array}{l}\mathrm{f}_{\text {sample }} \\
(\mathrm{kHz})\end{array}$ & $\begin{array}{c}\text { \# time } \\
\text { samples }\end{array}$ & $\begin{array}{c}\# \\
\text { averages }\end{array}$ & $\begin{array}{c}\text { point spacing } \\
\text { (mm) }\end{array}$ \\
\hline Coupon with QSID & 1 & 200 & 65 & $\begin{array}{l}50 \\
63 \\
70\end{array}$ & 100 & 1250 & 10000 & 10 & 1.3 \\
\hline $\begin{array}{l}\text { Coupon with } \\
\text { delaminations }\end{array}$ & 10 & 125 & 65 & 30 & 100 & 625 & 10000 & 7 & 1.5 \\
\hline Bicycle frame & 10 & 125 & 65 & $\begin{array}{l}30 \\
50\end{array}$ & 100 & 625 & 10000 & 10 & $\approx 1$ \\
\hline Aircraft panel & 10 & 125 & 65 & 30 & 100 & 625 & 10000 & 10 & 2.5 \\
\hline
\end{tabular}

The full wavefield velocity response is measured using a 3D SLDV (Polytec PSV-500-3D Xtra) with infrared measurement lasers. For each component, the scanned area is indicated on Figure 1. The scan point spacing, sample frequency and signal duration are listed in Table 1. Note that the scan point spacing equals the resolution of the obtained damage maps. In order to detect the nonlinear components, which are of very low amplitude $[30,38,40]$, removable reflective tape (3M type 680CRE10 ) is attached to the scan area. In addition, averages are taken to further increase the signal-to-noise ratio of the measurements (see Table 1 ). 


\section{Broadband NWMS - CFRP coupon with QSID}

The construction of a damage map consists of multiple steps which are schematically illustrated in Figure 2. Two major parts are differentiated, i.e. (i) nonlinear component extraction using timefrequency filtering and (ii) damage map construction using bandpower calculation. These two parts are discussed in detail after a short explanation of the working principle of the method which emphasizes the benefits of using the modulation sidebands compared to the higher harmonics.

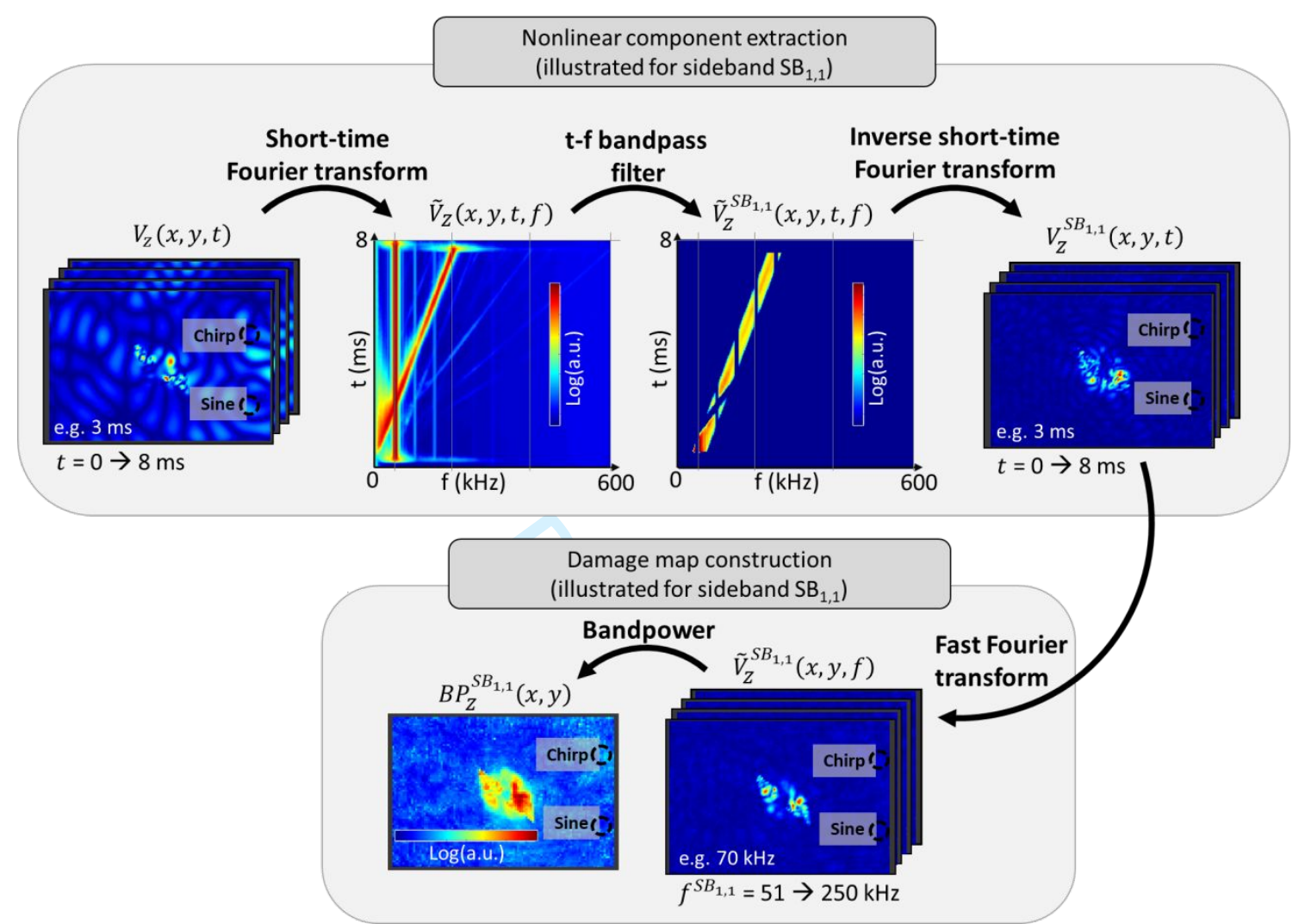

Figure 2: Workflow of the nonlinear broadband damage map construction algorithm.

\subsection{Working principle}

The interaction of elastic waves with a defect results in a non-classical nonlinear response of the defect due to contact and friction mechanisms [51]. Multiple extensive analytical [52, 53] and experimental reports [30] investigate the nature of this nonlinear behavior. Below, a short background is given assuming a clapping contact model (e.g. for delamination). This background illustrates the challenges in nonlinear inspection, and how to overcome them.

We consider the piezoelectric excitation of elastic waves in a delaminated composite component. When a sine voltage signal with frequency $f_{\text {in }}$ is supplied to the actuator, elastic waves are excited with frequency $f_{\text {in }}$. In addition, higher harmonic $(\mathrm{HH})$ components can be excited due to source nonlinearity. This is schematically illustrated in Figure 3 (a) where $f_{H_{i}}=i . f_{\text {in }}$ (with $i$ an integer) represent the higher harmonic frequency components with amplitudes $a_{H H_{i}}^{S}$. The superscript s indicates that these components are excited by the source. The source nonlinearity is a combination of nonlinearity present in the signal generator, the amplifier, the piezoelectric material and the bonding layer $[54,55]$. The piezoelectric excited waves interact with the nonlinear defect and again higher harmonic components can be formed. Thus, the resulting higher harmonics $\left(f_{H H_{i}}\right)$ are a combination of both source nonlinearity $\left(a_{H H_{i}}^{S}\right)$ as well as defect nonlinearity $\left(a_{H H_{i}}^{d}\right)$. Note that the $f_{H H_{i}}$ components which are present due to a nonlinear defect response to nonlinear components formed by the source are not included in the figure as they would be of an undetectable low amplitude $\left(a_{H H_{i}}^{S}\right.$. 
$\left.a_{H H_{i}}^{d}\right)$. As a result, when higher harmonic frequency components are detected, they could have been triggered by a nonlinear source, a nonlinear defect, or a combination of both.

In order to solve this problem, a second excitation source is added (see Figure 3 (b)). Each source is supplied with a different sine voltage signal (frequencies $f_{i n^{1}}, f_{i n^{2}}$ ). Again, higher harmonics of $f_{i n^{1}}$ and $f_{i n^{2}}$ are expected in the output signal which are partly caused by source $\left(a_{H H_{i}^{1}}^{S}\right.$ and $\left.a_{H H_{i}^{2}}^{S}\right)$ as well as by defect nonlinearity $\left(a_{H H_{i}}^{d}\right)$. In addition to the higher harmonics, modulation sidebands are formed exclusively due to defect nonlinearity. Only in case of a poorly bonded actuator, resulting in a partial disbond between actuator and component, sidebands can form at the location of the actuator. These components have frequency: $f_{S B_{i, j}}=i . f_{i n^{1}}+j . f_{i n^{2}}$ (with $i$ and $j$ integers) and amplitude $a_{S B_{i, j}}^{d}$. As such, the use of these modulation sidebands $\left(f_{S B_{i, j}}\right)$ instead of higher harmonics $\left(f_{H H_{i}}\right)$ overcomes the source nonlinearity problem and could lead to an improved damage map. This statement is experimentally verified throughout this manuscript for the first modulation sideband $\mathrm{SB}_{1,1}$ (written in bold in the schematic).

(a)

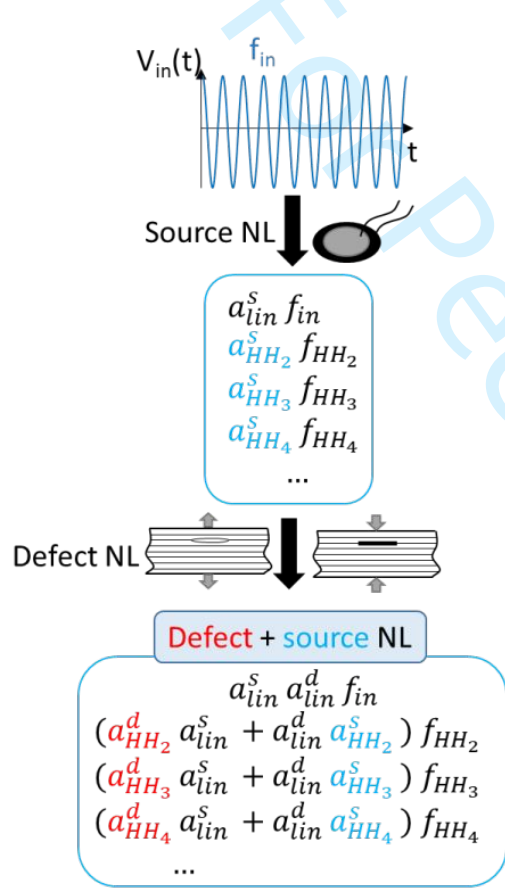

(b)
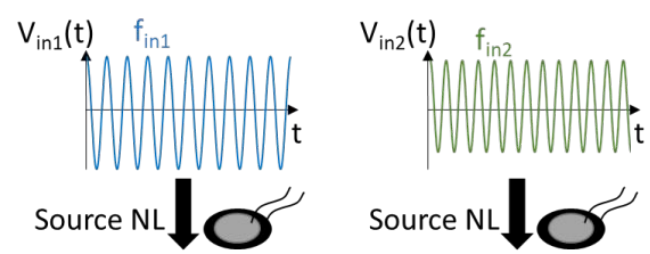

Source $\mathrm{NL}$
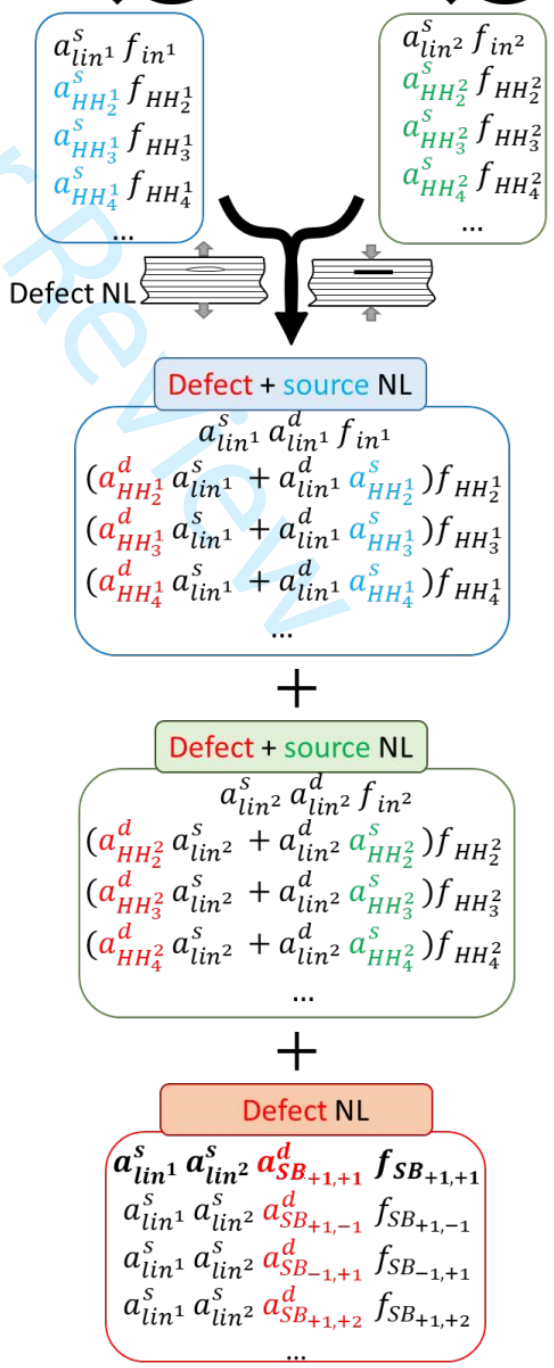

Figure 3: Schematic overview of the expected nonlinear components in a NEWS (a) and a NWMS (b) experiment. 
In order to activate contact acoustic nonlinearity, the vibrations at the defect must be of sufficient amplitude. Best is to match the excitation frequency with a LDR frequency. However, as this LDR frequency is not known a priori (depends on defect structure), a broadband excitation must be used. As such, one piezoelectric source is supplied with a broadband chirp voltage signal which triggers the nonlinear response of the defect at specific (LDR) frequencies. A second piezoelectric source is supplied with a sine voltage signal which results in the formation of the sideband frequency components used for the creation of the damage map.

\subsection{Nonlinear component extraction}

In a previous study of the current authors [38], it was illustrated how the short-time Fourier transform (STFT) and specific bandpass windows in the time-frequency domain can be used to extract higher harmonic components from broadband measurement data. Here, the same technique is used with the difference that there is a second sine excitation source present. The reader is referred to [38] for the details (e.g. all the formulas) of the filtering procedure and the associated equations.

The filtering procedure is illustrated using the measurement results of the CFRP coupon with QSID (see also Figure 2). First, the STFT is used to transform the response signal at each scan point from the time domain to the time-frequency domain. Figure 4 (a) shows the average of the resulting spectrograms and Figure 4 (d) shows the derived frequency spectrum corresponding to time instance $6.5 \mathrm{~ms}$. High intensity lines are visible in the spectrogram corresponding to: (i) the linear sine response $f_{\text {sine }}^{\text {lin }}$ at 50 $\mathrm{kHz}$, (ii) higher harmonics of the sine response e.g. $f_{\text {sine }}^{\mathrm{HH}_{2}}$ at $100 \mathrm{kHz}$, (iii) the linear chirp response $f_{\text {chirp }}^{\text {lin }}$ from 1 to $200 \mathrm{kHz}$, (iv) modulation sidebands e.g. first sideband $f_{S B_{1,1}}$ from $1+50=51 \mathrm{kHz}$ to $200+50$ $=250 \mathrm{kHz}$, and $(\mathrm{v})$ higher harmonics of the chirp response e.g. $f_{\text {chirp }}^{\mathrm{HH}_{2}}$ from 2 to $400 \mathrm{kHz}$.

In order to extract one of these components, a bandpass filter is constructed around the component of interest. As an example, the window shown in Figure $4(\mathrm{~b})$ is used to extract the $\mathrm{SB}_{1,1}$ sideband. Using this filter, all $\mathrm{SB}_{1,1}$ components are retained with the exception of the components which overlap in time-frequency domain with the higher harmonics of the sine and chirp excitation. This makes sure that no source nonlinearity is included in the filtered signal. For each scan point, the filter is multiplied with the local spectrogram. The average of all the $\tilde{V}_{Z}^{S B_{1,1}}$ filtered spectrograms is shown in Figure 4 (c). Finally, the filtered nonlinear component is obtained in time domain after inverse STFT.

The same filtering procedure is used for the extraction of the linear part of the chirp response $\tilde{V}_{Z}^{\text {lin }}$ and for the second order higher harmonic $\tilde{V}_{Z}^{H H_{2}}$ (see Figure 4 (e) and Figure 4 (f), respectively). 

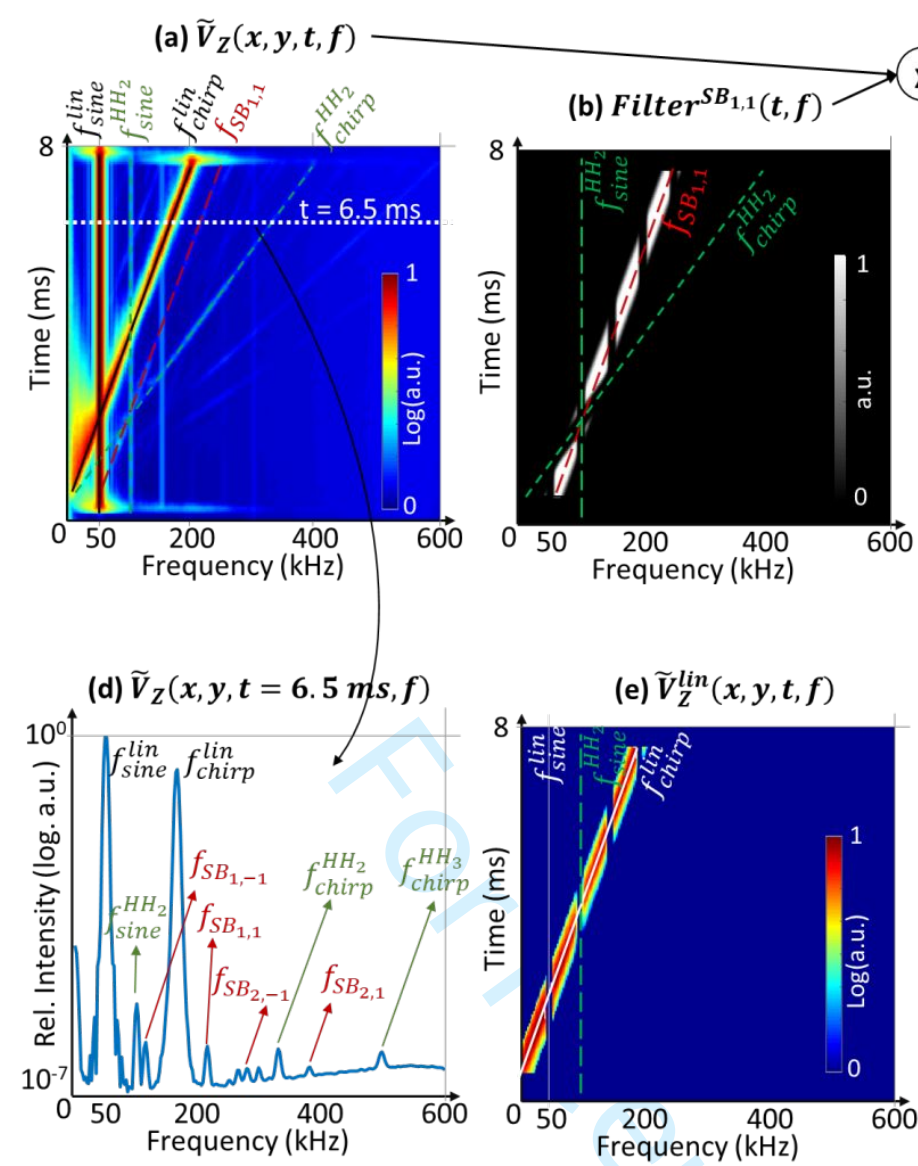<smiles>[CH]C1CCC1</smiles>

(c) $\widetilde{V}_{Z}^{S B_{1,1}}(x, y, t, f)$

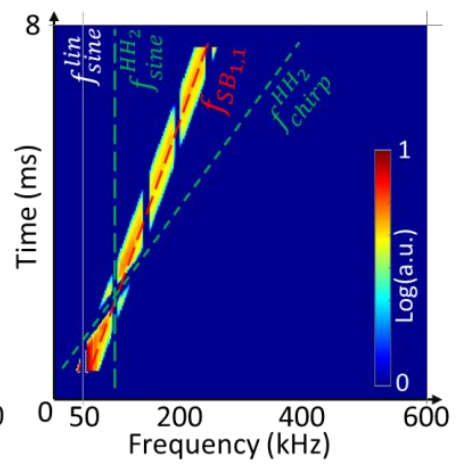

(e) $\widetilde{V}_{Z}^{\text {lin }}(x, y, t, f)$
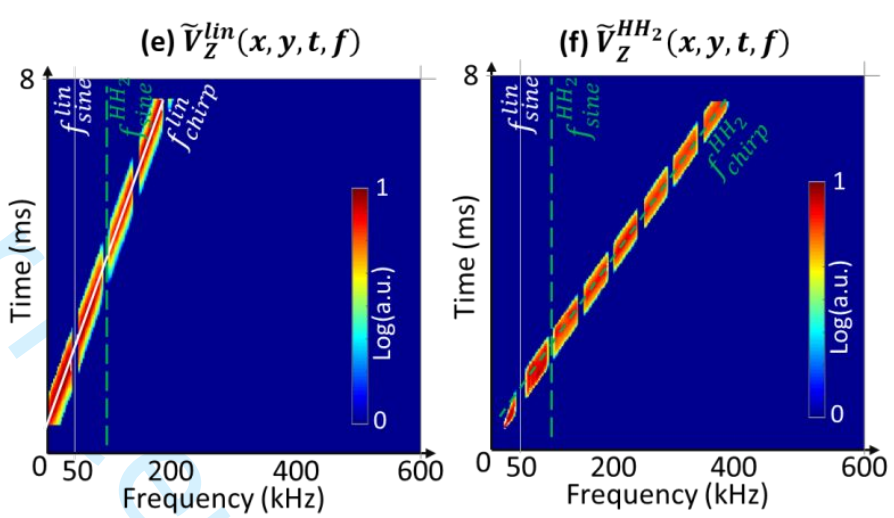

Figure 4: Extraction of specific velocity components: (a) Average spectrogram, (b) Bandpass filter for first sideband $\mathrm{SB}_{1,1}$ extraction, (c) Average spectrogram after $\mathrm{SB}_{1,1}$ extraction (d) Average frequency spectrum at time instance $6.5 \mathrm{~ms}$, (e) Average spectrogram after linear component extraction and (f) Average spectrogram after second order higher harmonic $\left(\mathrm{HH}_{2}\right)$ extraction.

\subsection{Bandpower calculation}

Figure 5 shows the amplitude of vibration averaged over the area of the chirp actuator (blue curve), the defect (red curve) and the damage-free material (black curve) for: (a) the linear chirp response $\tilde{V}_{Z}^{l i n}$, (b) the second higher harmonic response $\tilde{V}_{Z}^{H H_{2}}$ and (c) the first modulation sideband $\tilde{V}_{Z}^{S B_{1,1}}$. The amplitude axis is normalized and shown in logarithmic scale. The amplitude reduction at 50,100, 150 ... $\mathrm{kHz}$ is attributed to the bandstop filter at the sine excitation frequency and its higher harmonics (see previous section).

The linear response (Figure 5 (a)) shows a lower intensity at the damage-free material compared to at the location of the chirp actuator. This is the result of material damping, especially in the higher frequency range, and geometric wave spreading. The linear response at the defect is higher than at the background for the majority of frequencies which is caused by the reduced bending stiffness at the defect. Such an increased intensity of the linear component would not be present in case of deeper defects (see [14] and also Section 4.1).

The curves for the second harmonic response (Figure 5 (b)) show that $\mathrm{HH}_{2}$ components are formed both at the defect and at the actuator. The defect nonlinearity is dominant between 50 and $150 \mathrm{kHz}$ whereas the source nonlinearity dominates in the higher frequency regime. The damage-free material shows only minor indications of nonlinearity.

The first modulation sideband amplitude (Figure 5 (c)) at the chirp actuator is low and similar to the amplitude measured in damage-free material. This confirms that this modulation component is not formed at the actuator. The sideband amplitude is considerably higher at the location of the defect over the entire frequency range. As such, it is confirmed that the use of this sideband component is 
advantageous as it is exclusively formed due to the presence of a defect (and not due to source nonlinearity).
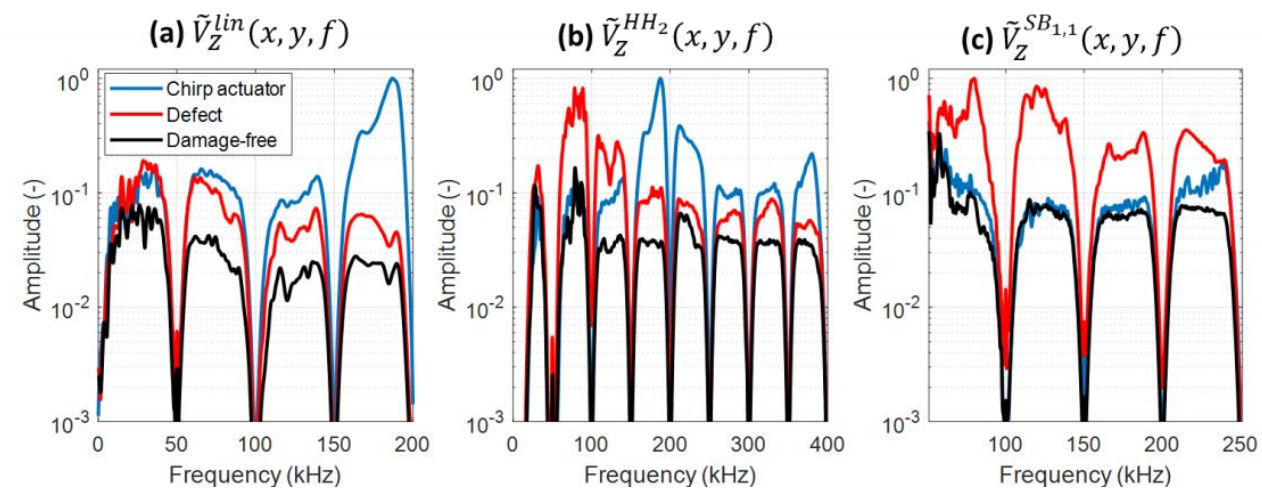

Figure 5: Vibrational intensity at the chirp actuator (blue curve), the damage-free material (black curve) and the defect (red curve): (a) linear response, (b) second order harmonic response and (c) first modulation sideband.

A damage map is derived from each of the extracted velocity components using broadband bandpower (BP) calculation:

$$
B P_{Z}^{*}\left(x, y, f\left(k_{1}\right), f\left(k_{2}\right)\right)=\frac{1}{k_{2}-k_{1}} \sum_{k=k_{1}}^{k_{2}} \tilde{V}_{Z}^{*}(x, y, f(k))^{2}
$$

The bandpower $B P_{Z}^{*}$ gives the vibrational energy at the point with location $(x, y)$ related to velocity component $\tilde{V}_{Z}^{*}$ for a frequency band from $f\left(k_{1}\right)$ up to $f\left(k_{2}\right)$. The variable $k$ is the frequency index and $1 \leq k_{1}<k_{2} \leq \frac{\# \text { samples }}{2}$. The velocity component $\tilde{V}_{Z}^{*}$ can be any of the filtered signals: $\tilde{V}_{Z}^{l i n}, \tilde{V}_{Z}^{H H_{2}}, \tilde{V}_{Z}^{S B_{1,1}}$ (as indicated by the *). Note that it is also possible to use the in-plane vibrational component $\left(\tilde{V}_{x}^{*}, \tilde{V}_{y}^{*}\right)$ as input which can be beneficial to detect defects with out-of-plane interfaces such as cracks [12].

The resulting bandpower maps are scaled according to their maximum and are shown in Figure 6 using a logarithmic colorscale. For each velocity component, the total relevant frequency range was used for the calculation of the damage map. A defect-to-background ratio (DBR) is introduced as the ratio between the average intensity at the defect and the average intensity at the damage-free material (i.e. the total scan area excluding the defect area, but including the actuator area). As such, a DBR of 1 corresponds to an absence of the defect in the damage-map while the defect detection improves for higher DBRs. The $B P_{Z}^{*}$ is related to the integral of the curves shown in Figure 5. As a result, the damage maps reflect the same observations made in the analysis of Figure 5.

The $B P_{Z}^{\text {lin }}$ map (Figure $6(\mathrm{a})$ ) shows the presence of the QSID with a limited contrast compared to the damage-free material ( $D B R=2$ ). The contrast at the damage is largely improved when using nonlinear components. For the $\mathrm{BP}_{Z}^{\mathrm{HH}_{2}}$ map (Figure 6 (b)), a pronounced amplitude increase at the QSID is observed leading to a DBR of 18 . However, also the source nonlinearity is captured in this map. The $B$ $P_{Z}^{S B_{1,1}}$ map (Figure 6 (c)) shows the best damage map performance with a DBR of 47 and no influence of the source nonlinearity. 


\subsection{Effect of sine excitation frequency}

It is well known that the nonlinear response of a defect is highly dependent on the amplitude of vibrations at the defect. Chirping the excitation signal of one source over a large frequency band ensures that several LDR frequencies of the defect are excited, resulting in the required high amplitude of vibration at the defect. The second actuator is then used to generate sideband components in order to exclude the effect of source nonlinearity. A second important benefit of adding the secondary sine excitation is the additional increase of the amplitude of vibration at the defect. In order to investigate this further, the experiment is repeated twice using a different sine excitation frequency than the previously used $50 \mathrm{kHz}$ : (i) another arbitrary frequency of $70 \mathrm{kHz}$ and (ii) a LDR frequency of $63 \mathrm{kHz}$. The operational deflection shapes at these sine frequencies $(50 \mathrm{kHz}, 63 \mathrm{kHz}$ and $70 \mathrm{kHz})$ are shown in Figure 7 (a-c). For all three cases, there is energy trapping at the defect leading to an increased vibrational amplitude. For the frequency of $63 \mathrm{kHz}$, the amplitude increase is the largest and occurs at a small area of the BVID which is indicated with LDR on Figure 7 (b).

Figure 7 (d-e) show the resulting $\mathrm{SB}_{1,1}$ bandpower maps (with identical logarithmic colorscale). The highest DBR ratio (and thus the best damage map) is obtained for the case that the sine excitation frequency matches a LDR frequency (Figure $7(b, e)$ ). The maximum in the bandpower map also matches well with the fraction of the defect showing the LDR behavior. The nonlinear response of the defect is high enough to make it behave as a source of nonlinear components. This source behavior is seen by the wave-like increased intensity of the bandpower in the damage-free material close to the defect.

In a real NDT experiment, there is no information on the defects (if any) and it is impossible to match the sine excitation frequency with a LDR. As a result, an arbitrary sine frequency is used in the remainder of the study. It is advised to use a frequency which results in elastic waves which have a spatial wavelength smaller than the size of the defects to be found. In this way, multiple wavelengths 
fit inside the defect resulting in an increased local amplitude (due to wave energy trapping) and potentially a LDR $[6,7]$. For instance for this $5.5 \mathrm{~mm}$ thick CFRP coupon with QSID, the sine excitation frequencies $50 \mathrm{kHz}, 63 \mathrm{kHz}$ and $70 \mathrm{kHz}$ are associated with a damage-free wavelength of $23 \mathrm{~mm}, 19$ $\mathrm{mm}$ and $18 \mathrm{~mm}$, respectively. At the defect, the wavelength is even smaller due to the lower thickness associated to the delaminations. As a result, the wavelengths at the defect are indeed smaller than the defect's width of $\pm 34 \mathrm{~mm}$. The resulting energy trapping at the QSID was already confirmed by Figure 7 (a-c).
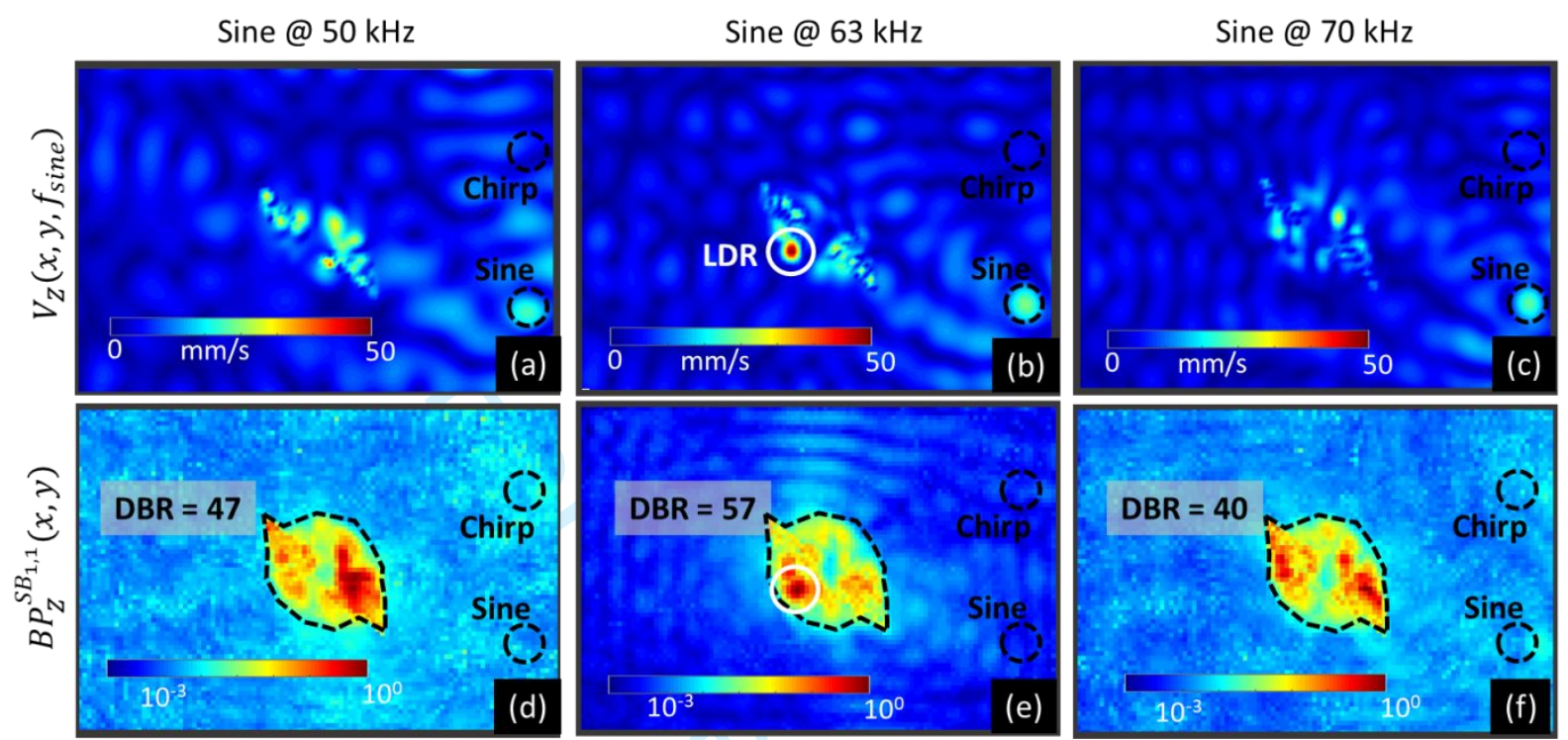

Figure 7: Effect of sine frequency on broadband nonlinear bandpower damage map: (a-c) Operational deflection shape at the frequency of the sine excitation, (d-f) Bandpower maps of the first modulation sideband.

\section{Results of broadband NWMS - Other test specimens}

\subsection{CFRP coupon with artificial delaminations}

The processing steps discussed in the previous section are repeated for the measurement results of the CFRP coupon with one shallow and one deep artificial delamination (see Figure 1 (b)). Three potential damage maps are constructed using bandpower calculation: for the linear part of the velocity response, for the second higher harmonic and for the first spectral sideband. These maps are shown in Figure 8 (using an identical logarithmic color scale) together with the operational deflection shape at the arbitrarily selected sine excitation frequency of $30 \mathrm{kHz}$. 

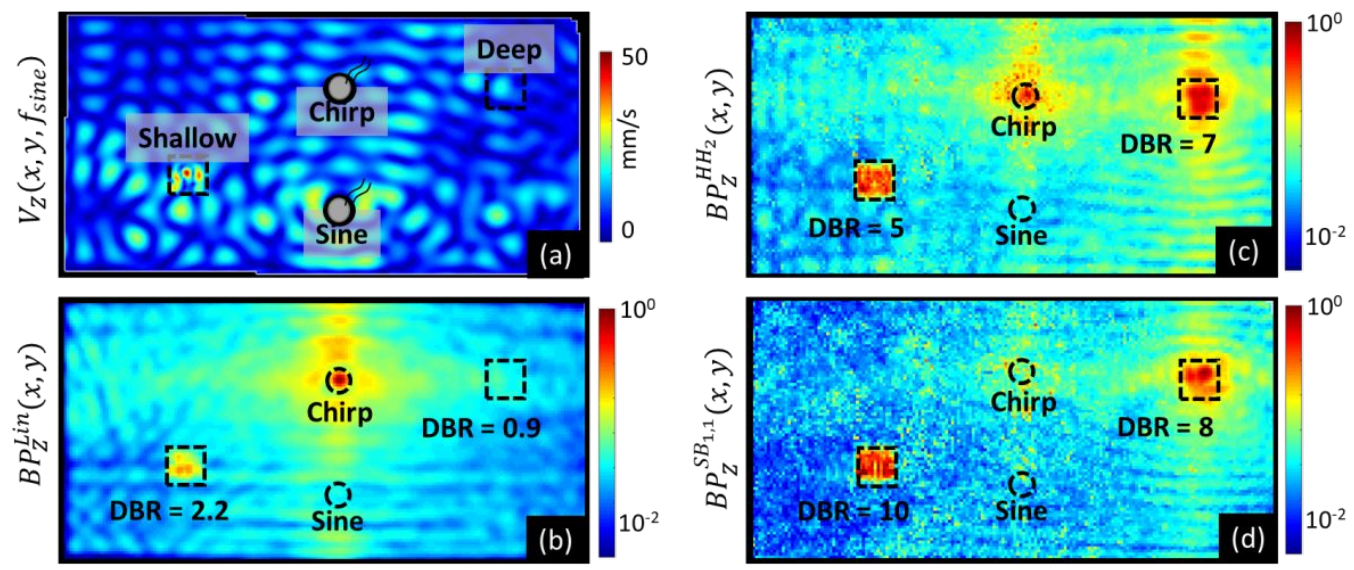

Figure 8: CFRP coupon with a shallow and deep delamination: (a) Operational deflection shape at sine excitation frequency $f_{\text {sine }}=30 \mathrm{kHz}$, and Bandpower of (b) linear response 10 to $125 \mathrm{kHz}$, (c) second order higher harmonic response 20 to $250 \mathrm{kHz}$ and (d) first order modulation sideband in 40 to $155 \mathrm{kHz}$.

The arbitrary selected sine frequency of $30 \mathrm{kHz}$ does not match with an LDR. However, there is still an increased amplitude of vibration observed at the shallow delamination thanks to wave energy trapping (see Figure $8(a)$ ). The pronounced stiffness reduction at the shallow delaminations makes it visible $(D B R=2.2)$ in the $B P_{Z}^{l i n}$ map (see Figure $\left.8(b)\right)$. The deep (backside) delamination is associated with a limited reduction in the bending stiffness (when looking from the scan side) which makes it invisible (DBR $=0.9)$ in the linear response.

The nonlinear bandpower maps reveal both the shallow and the deep delaminations, with a higher DBR for the shallow delamination. The sideband bandpower map (Figure 8 (d)) is superior compared to the second higher harmonic bandpower map (Figure 8 (c)). This is seen by the increase in DBR at both defect locations. In addition, the sideband bandpower map is not affected by source nonlinearity.

\subsection{Structural CFRP test specimens}

Structural components made out of CFRP are often curved, have anisotropic material properties and may include add-ons such as stiffeners. Here, the robustness of the proposed nonlinear damage map construction method is verified using the measurement results of a bicycle frame down tube and a stiffened aircraft panel.

Two measurements are performed for the bicycle down tube with sine excitation frequencies $30 \mathrm{kHz}$ and $50 \mathrm{kHz}$. The overview of the results is shown in Figure 9. The curvature of the test specimen made it impossible to quantify the extent of the damage using our ultrasonic C-scan setup. As a result, the estimate of the extent of the damage (as indicated by the black ellipse) is based on the outcome of the sideband bandpower (i.e. Figure $9(e, j)$, see further).

The operational deflection shapes at the sine excitation frequencies are shown in Figure 9 (a) and (f). A limited increase in wave amplitude is observed at the location of the defect due to energy trapping. However, it is clear that these operational deflection shapes cannot be used for damage detection. The bandpower maps of the linear response (Figure $9(b, g)$ ) and the bandpower maps of the second higher harmonic component (Figure $9(\mathrm{c}, \mathrm{h})$ ) cannot be used for damage identification either as the chirp actuators introduce a large amount of linear and nonlinear components which cover almost the whole measurement domain. The relatively high amount of source nonlinearity captured in the $B P_{Z}^{\mathrm{HH}_{2}}$ maps is presumably caused by the geometric mismatch between the flat piezoelectric actuators and the tubular frame. In contrast, the location of the BVID is successfully revealed using the bandpower maps calculated for the first modulation sideband (see Figure $9(d, i)$ ). Note that the use of a linearly scaled colorbar further highlights this high intensity difference between BVID and damage-free material (see Figure $9(\mathrm{e}, \mathrm{j})$ ). A higher DBR is obtained when using a $50 \mathrm{kHz}$ sine excitation (i.e. DBR = 23) compared to a $30 \mathrm{kHz}$ sine excitation (i.e. $\mathrm{DBR}=9$ ). This is related to the superior wave energy 
trapping at $50 \mathrm{kHz}$ compared to $30 \mathrm{kHz}$ as seen in the corresponding operational deflection shapes (Figure $9(a, f)$ ).

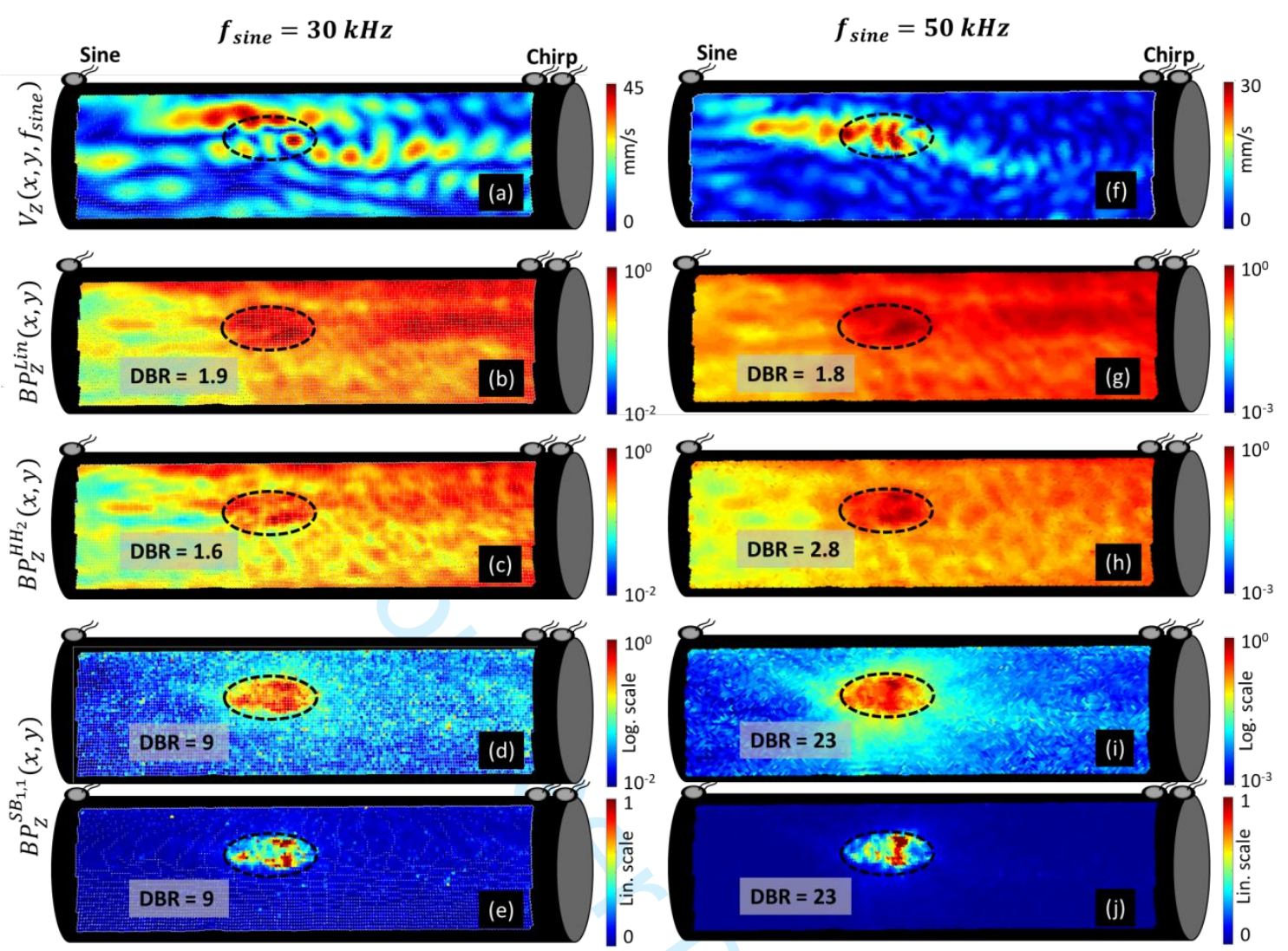

Figure 9: Impact damage detection in a CFRP bicycle frame down tube: (a,f) Operational deflection shape at sine excitation frequency, and Bandpower of (b,g) linear response 10 to $125 \mathrm{kHz},(\mathrm{c}, \mathrm{h})$ second order higher harmonic response 20 to 250 $\mathrm{kHz}$ and (d-e,i-j) first order modulation sideband with logarithmic and linear color scale, respectively. (a-e) $30 \mathrm{kHz}$ sine excitation, (f-j) $50 \mathrm{kHz}$ sine excitation.

The results for the CFRP aircraft panel are shown in Figure 10 for a sine excitation frequency equal to $30 \mathrm{kHz}$. The operational deflection shape (Figure 10 (a)) has a chaotic appearance, and gives no indication of damage. The defect is marginally visible in the linear bandpower map (Figure 10 (b)). Only at the location of the shallow delamination, the intensity is higher compared to the intensity of the virgin left-side of the stiffener. The damaged area becomes visible as a zone of increased intensity in the higher harmonic bandpower map (Figure 10 (c)). However, the $B P_{Z}^{\mathrm{HH}_{2}}$ map is again polluted by source nonlinearity resulting in the limited $D B R=2$. The bandpower map of the first spectral sideband (Figure 10 (d)) are found to exclusively reveal the damage with a satisfying DBR of 11 . The pronounced increase in intensity is further disclosed when using a linearly scaled colorbar (Figure 10 (e)). 


\section{Conclusions}

A full-field inspection method, based on nonlinear elastic wave modulation spectroscopy (NWMS), is proposed for damage detection and assessment in typical CFRP parts in a baseline-free and userindependent manner. The procedure results in a unique damage map that only requires a single experiment, uses low power piezoelectric actuators, is not affected by source nonlinearity, and is applicable to complex structural CFRP components.

The test specimen are excited using two low power piezoelectric actuators. One actuator is supplied with a broadband chirp voltage signal to make sure that LDR frequencies of the defects are excited. This is required to achieve a high enough vibrational amplitude to trigger the nonlinear response at the defect. The second actuator is supplied with a sine voltage signal. Nonlinear vibrational components are formed such as higher harmonics of both the sine and the chirp excitation, as well as modulation sidebands.

A bandpass filtering method in the time-frequency domain is proposed for extracting specific linear as well as nonlinear components from the measurement data. The amplitudes of the linear part of the 
response, the second higher harmonic and the first modulation sideband are investigated. In contrast to the linear response, the nonlinear components show a high intensity at the (deep) defects. Though, the higher harmonic response experiences quite some negative effects from source nonlinearity, partially masking the sought-after defect nonlinearity. It is only the modulation sideband which shows high performance in highlighting defects as it is almost not affected by source nonlinearity. Based on these observations, damage maps are constructed using broadband bandpower calculation.

The damage sensitivity of the broadband bandpower maps is verified using the measurement results of four test specimens: (i) a coupon with QSID, (ii) a coupon with one shallow and one deep delamination, (iii) an impacted down tube of a bicycle frame and (iv) an aircraft panel with damage at a backside stiffener. In all investigated cases, the broadband bandpower map of the first modulation sideband was highly effective in revealing the defects.

\section{Acknowledgments and funding}

The authors acknowledge Fonds voor Wetenschappelijk Onderzoek FWO (fellowships 1148018N, $1 \mathrm{~S} 11520 \mathrm{~N}$ and $12 \mathrm{T5418N}$ ) and the SBO project DETECT-IV (Grant no. 160455), which fits in the SIM research program MacroModelMat (M3) coordinated by Siemens (Siemens Digital Industries Software, Belgium) and funded by SIM (Strategic Initiative Materials in Flanders) and VLAIO (Flemish government agency Flanders Innovation \& Entrepreneurship). The authors further thank Honda and SABCA Limburg for providing the CFRP coupon material and the CFRP aircraft panel, receptively.

\section{Declaration of conflicting interests}

The authors declared no potential conflicts of interest with respect to the research, authorship and/or publication of this article.

\section{References}

1. Mei, H., et al., Recent Advances in Piezoelectric Wafer Active Sensors for Structural Health Monitoring Applications. Sensors (Basel, Switzerland), 2019. 19(2).

2. Jurek, M., et al., Non-contact excitation and focusing of guided waves in CFRP composite plate by air-coupled transducers for application in damage detection. Procedia Structural Integrity, 2018. 13: p. 2089-2094.

3. Davies, S.J., et al., Laser-generated ultrasound: its properties, mechanisms and multifarious applications. Journal of Physics D: Applied Physics, 1993. 26(3): p. 329-348.

4. Solodov, I., A. Dillenz, and M. Kreutzbruck, A new mode of acoustic NDT via resonant aircoupled emission. Journal of Applied Physics, 2017. 121(24).

5. Ostachowicz, W., M. Radzieński, and P. Kudela, 50th Anniversary Article: Comparison Studies of Full Wavefield Signal Processing for Crack Detection. Strain, 2014. 50(4): p. 275-291.

6. Glushkov, E., et al., Wave energy trapping and localization in a plate with a delamination. Smart Materials and Structures, 2012. 21(12).

7. Munian, R.K., D.R. Mahapatra, and S. Gopalakrishnan, Lamb wave interaction with composite delamination. Composite Structures, 2018. 206: p. 484-498.

8. Wojtczak, E. and M. Rucka, Wave Frequency Effects on Damage Imaging in Adhesive Joints Using Lamb Waves and RMS. Materials (Basel, Switzerland), 2019. 12(11).

9. Radzieński, M., et al., Application of RMS for damage detection by guided elastic waves. Journal of Physics: Conference Series, 2011. 305.

10. Kudela, P., et al., Application of scanning laser Doppler vibrometry for delamination detection in composite structures. Optics and Lasers in Engineering, 2017. 99: p. 46-57. 
11. Solodov, I., et al., A local defect resonance to enhance acoustic wave-defect interaction in ultrasonic nondestructive evaluation. Applied Physics Letters, 2011. 99(21).

12. Segers, J., et al., Towards in-plane local defect resonance for non-destructive testing of polymers and composites. NDT \& E International, 2018. 98: p. 130-133.

13. Segers, J., et al., Efficient automated extraction of local defect resonance parameters in fiber reinforced polymers using data compression and iterative amplitude thresholding. Journal of Sound and Vibration, 2019. 463.

14. Segers, J., et al., Probing the limits of full-field linear Local Defect Resonance identification for deep defect detection. Ultrasonics, 2020. 105.

15. Jeon, J.Y., et al., Damage detection on composite structures with standing wave excitation and wavenumber analysis. Advanced Composite Materials, 2017. 26(sup1): p. 53-65.

16. Flynn, E.B., et al., Structural imaging through local wavenumber estimation of guided waves. NDT \& E International, 2013. 59: p. 1-10.

17. Yu, L., Z. Tian, and C.A. Leckey, Crack imaging and quantification in aluminum plates with guided wave wavenumber analysis methods. Ultrasonics, 2015. 62: p. 203-12.

18. Mesnil, O., C.A.C. Leckey, and M. Ruzzene, Instantaneous and local wavenumber estimations for damage quantification in composites. Structural Health Monitoring, 2014. 14(3): p. 193204.

19. Rogge, M.D. and C.A. Leckey, Characterization of impact damage in composite laminates using guided wavefield imaging and local wavenumber domain analysis. Ultrasonics, 2013. 53(7): p. 1217-26.

20. Gao, T., et al., Hidden Corrosion Detection Using Laser Ultrasonic Guided Waves with Multifrequency Local Wavenumber Estimation. Ultrasonics, 2020. 108.

21. O'Dowd, N.M., et al., Exploring the Performance Limits of Full-Field Acoustic Wavenumber Spectroscopy Techniques for Damage Detection through Numerical Simulation, in 8th European Workshop on Structural Health Monitoring. 2016, NDT.net: Bilbao Spain.

22. Moon, S., et al., Optimization of excitation frequency and guided wave mode in acoustic wavenumber spectroscopy for shallow wall-thinning defect detection. Journal of Mechanical Science and Technology, 2018. 32(11): p. 5213-5221.

23. Ruzzene, M., Frequency-wavenumber domain filtering for improved damage visualization. Smart Materials and Structures, 2007. 16(6): p. 2116-2129.

24. Girolamo, D., H.Y. Chang, and F.G. Yuan, Impact damage visualization in a honeycomb composite panel through laser inspection using zero-lag cross-correlation imaging condition. Ultrasonics, 2018. 87: p. 152-165.

25. Yu, X., et al., Detection of damage in welded joints using high order feature guided ultrasonic waves. Mechanical Systems and Signal Processing, 2019. 126: p. 176-192.

26. Radzienski, M., et al., Damage Identification in Various Types of Composite Plates Using Guided Waves Excited by a Piezoelectric Transducer and Measured by a Laser Vibrometer. Sensors (Basel, Switzerland), 2019. 19(9).

27. Kudela, P., M. Radzieński, and W. Ostachowicz, Identification of cracks in thin-walled structures by means of wavenumber filtering. Mechanical Systems and Signal Processing, 2015. 50-51: p. 456-466.

28. Kudela, P., M. Radzienski, and W. Ostachowicz, Impact induced damage assessment by means of Lamb wave image processing. Mechanical Systems and Signal Processing, 2018. 102: p. 2336.

29. Segers, J., et al., Robust and baseline-free full-field defect detection in complex composite parts through weighted broadband energy mapping of mode-removed guided waves. Mechanical Systems and Signal Processing, 2020. Accepted for publication.

30. Jhang, K.-Y., et al., Measurement of Nonlinear Ultrasonic Characteristics. Measurement Science and Technology, ed. M.G. Cain, et al. 2020: Springer. 
31. Van Den Abeele, K., et al., Nonlinear Elastic Wave Spectroscopy (NEWS) Techniques to Discern Material Damage, Part II: Single-Mode Nonlinear Resonance Acoustic Spectroscopy. Res Nondestr Eval 2000. 12: p. 31-42.

32. Zhang, S., et al., Far-sided defect recognition of FRP sandwich structures based on local defect resonance. Journal of Sandwich Structures \& Materials, 2019: p. 1-12.

33. Fierro, G.P.M. and M. Meo, Nonlinear elastic imaging of barely visible impact damage in composite structures using a constructive nonlinear array sweep technique. Ultrasonics, 2018. 90: p. 125-143.

34. Fierro, G.P.M. and M. Meo, A combined linear and nonlinear ultrasound time-domain approach for impact damage detection in composite structures using a constructive nonlinear array technique. Ultrasonics, 2018. 93: p. 43-62.

35. Ginzburg, D., Damage Propagation and Detection using Nonlinear Elastic Wave Spectroscopy in Aerospace Structures, in Department of Mechanical Engineering. 2016, University of Bath.

36. Ooijevaar, T.H., et al., Nonlinear dynamic behavior of an impact damaged composite skinstiffener structure. Journal of Sound and Vibration, 2015. 353: p. 243-258.

37. Broda, D., et al., Modelling of nonlinear crack-wave interactions for damage detection based on ultrasound-A review. Journal of Sound and Vibration, 2014. 333(4): p. 1097-1118.

38. Segers, J., et al., Nonlinear Elastic Wave Energy Imaging for the Detection and Localization of In-Sight and Out-of-Sight Defects in Composites. Applied Sciences, 2020. 10(3924).

39. Su, Z., et al., Acousto-ultrasonics-based fatigue damage characterization: Linear versus nonlinear signal features. Mechanical Systems and Signal Processing, 2014. 45(1): p. 225-239.

40. Segers, J., et al., Backside delamination detection in composites through local defect resonance induced nonlinear source behaviour. Journal of Sound and Vibration, 2020. 479.

41. Van Den Abeele, K., P.A. Johnson, and A. Sutin, Nonlinear Elastic Wave Spectroscopy (NEWS) Techniques to Discern Material Damage, Part I: Nonlinear Wave Modulation Spectroscopy (NWMS). Res Nondestr Eval 2000. 12: p. 17-30.

42. Pieczonka, L., et al., Damage imaging in composites using nonlinear vibro-acoustic wave modulations. Structural Control and Health Monitoring, 2017.

43. Dziedziech, K., et al., Efficient swept sine chirp excitation in the non-linear vibro-acoustic wave modulation technique used for damage detection. Structural Health Monitoring, 2017. 17(3): p. 565-576.

44. Klepka, A., et al., Impact damage detection in composite chiral sandwich panels using nonlinear vibro-acoustic modulations. Smart Materials and Structures, 2013. 22(8).

45. Mevissen, F. and M. Meo, A Nonlinear Ultrasonic Modulation Method for Crack Detection in Turbine Blades. Aerospace, 2020. 7(6).

46. Fierro, G.P.M. and M. Meo, IWSHM 2017: Structural health monitoring of the loosening in a multi-bolt structure using linear and modulated nonlinear ultrasound acoustic moments approach. Structural Health Monitoring, 2018. 17(6): p. 1349-1364.

47. Klepka, A., et al., Nonlinear acoustics for fatigue crack detection - experimental investigations of vibro-acoustic wave modulations. Structural Health Monitoring, 2011. 11(2): p. 197-211.

48. Sohn, H., et al., Nonlinear ultrasonic wave modulation for online fatigue crack detection. Journal of Sound and Vibration, 2014. 333(5): p. 1473-1484.

49. Malfense Fierro, G.P., F. Ciampa, and M. Meo, Phase symmetry analysis for nonlinear ultrasonic modulated signals. Structural Control and Health Monitoring, 2020. 27(5).

50. Spronk, S.W.F., et al., Comparing damage from low-velocity impact and quasi-static indentation in automotive carbon/epoxy and glass/polyamide-6 laminates. Polymer Testing, 2018. 65: p. 231-241.

51. Hirsekorn, S. and P.P. Delsanto, On the universality of nonclassical nonlinear phenomena and their classification. Applied Physics Letters, 2004. 84(8): p. 1413-1415.

52. Ciampa, F., G. Scarselli, and M. Meo, On the generation of nonlinear damage resonance intermodulation for elastic wave spectroscopy. The Journal of the Acoustical Society of America, 2017. 141(4): p. 2364. 
53. Solodov, I., N. Krohn, and G. Busse, CAN: an example of nonclassical acoustic nonlinearity in solids. Ultrasonics, 2002. 40(1): p. 621-625.

54. Sun, L., et al., Technique to minimize couplant-effect in acoustic nonlinearity measurements. The Journal of the Acoustical Society of America, 2006. 120(5): p. 2500-2505.

55. Arafa, M. and A. Baz, On the Nonlinear Behavior of Piezoelectric Actuators. Journal of Vibration and Control, 2016. 10(3): p. 387-398. 